\title{
Multi-beam Physical Security Scheme: Security Assessment and Impact of Array Impairments on Security and Quality of Service
}

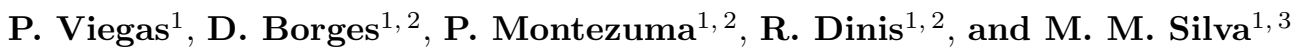 \\ ${ }^{1}$ IT, Instituto de Telecomunicações, Av. Rovisco Pais, Lisboa, Portugal \\ ${ }^{2}$ DEE, FCT Universidade Nova de Lisboa, Portugal \\ ${ }^{3}$ Universidade Autonoma de Lisboa, Portugal
}

\begin{abstract}
Massive multiple-input multiple-output (mMIMO) with perfect channel state information (CSI) can lead array power gain increments proportional to the number of antennas. Despite this fact constrains on power amplification still exist due to envelope variations of high order constellation signals. These constrains can be overpassed by a transmitter with several amplification branches, with each one associated to a component signal that results from the decomposition of a multilevel constellation as a sum of several quasi constant envelope signals that are sent independently. When combined with antenna arrays at the end of each amplification branch the security improves due to the energy separation achieved by beamforming. However, to avoid distortion on the signal resulting from the combination of all components at channel level all the beams of signal components should be directed in same direction. In such conditions it is crucial to assess the impact of misalignments between beams associated to each user, which is the purpose of this work. The set of results presented here show the good tolerance against misalignments of these transmission structures.
\end{abstract}

\section{INTRODUCTION}

Due to the demand of higher transmission rates, millimetric waves (mmWave) will be employed by future mobile wireless systems $[1,2]$. As example we can name the next generation of cellular systems, $5 \mathrm{G}$, that will use the spectrum of long term evolution (LTE) in frequency range from $600 \mathrm{MHz}$ to $6 \mathrm{GHz}$ and also the mmWave band $(24$ to $86 \mathrm{GHz})$. The use of mmWave bands opens a new door for massive MIMO (mMIMO) implementations both in base station and mobile terminals due to small dimensions antennas needed for this bands. Besides the reduction in the transmitted power allowed by mMIMO implementations, the base station throughput could be also improved by massive deployments of multiplexing techniques [1]. Notwithstanding, the deployment of a very high number of antennas for the maximization of spectral efficiency also implies the resort to high order constellations or multicarrier modulations such as orthogonal frequency digital multiplexing (OFDM), well known by the high peak-to-average power ratio (PAPR), which compromises power amplification's efficiency. The use of millimetric waves (mmWaves) also paves the way for the implementation of a multi layered transmission structure, with a first layer composed by a multibranch amplification structure, a second layer composed by the antenna array connected to each one amplifier's output and a third layer associated to spatial multiplexing that is composed by several layers 1 and 2 in parallel [3]. First layer of such structure employs a decomposition of multilevel constellation symbols as a sum of polar components, that can be modulated as bi-phase shift keying (BPSK) or as quadrature PSK (QPSK) components with different amplitudes that are amplified by a nonlinear (NL) power amplifier [45]. Under these conditions the original constellation with high PAPR is decomposed into components with lower PAPR that are amplified by the amplifier of each branch and sent to the channel. Since the bit streams in different branches are uncorrelated the overall radiation pattern of the antennas connected to the first layer remains omnidirectional. Although, a information directivity is introduced in the transmitted symbol since the components may suffer different rotation phases according the azimuthal direction. This also means that energy directivity is only due the contribution of layer 2, that performs beamforming.

The use of mmWaves means also higher transmission bandwidths and consequently a frequency selectivity behavior of the channel. Having in mind this impairment it is adopted a single-carrier with frequency domain equalization (SC-FDE) scheme [6,7].

It was already shown that these transmitters have same robustness against interference than transmitters with common beamforming (based on a 2-D array) without penalties on system's performance [8]. However, since the constellation symbol is obtained at channel level all the beams 
should be aligned when layer 2 is also employed. Having in mind previous considerations, it seems critical to study the alignments' requirements for the beams and the impact of misalignments between the beams carrying the signals components of each user in this multi layered structure, which is the purpose of this paper. This rest of the paper is organized as follows: Section 2 characterizes the layered transmitter structure. The characterization of the system is done in Section 3. The assessment of the impact of imperfect beam alignment is analyzed in Section 4. Finally, Section 5 summarizes the paper.

\section{MULTI LAYERED TRANSMITTER ARCHITECTURE}

It was already shown that multilevel constellations can be decomposed in polar components with the constellation symbols expressed as a function of the corresponding bits [9]. Let $\mathcal{S}=\left\{s_{0}, s_{1}, \ldots\right.$, $\left.\left.s_{(} N-1\right)\right\}$, a constellation with $M$ points (i.e., $\# \mathcal{S}=M$ ), where $s_{n} \in \mathbb{C}$. To each constellation point $s_{n}$ we associate a set of $\mu=\log _{2}(M)$ bits in polar format $\mathcal{B}=\left\{b_{n}^{0}, b_{n}^{1}, \ldots, b_{n}^{(\mu-1)}\right\}$ (i.e., $b_{n}^{(i)}= \pm 1=2 \beta_{n}^{(i)}-1, \beta_{n}^{(i)}=0$ or 1$)$. The set of $\mu$ bits can be decomposed in $M=2^{\mu}$ different subsets $\mathcal{B}_{m}, m=0,1, \ldots, M-1$.

Since we have $M$ constellation points in $\mathcal{S}$ and $M$ different subsets of $\mathcal{B}, \mathcal{B}_{0}, \mathcal{B}_{1}, \ldots, \mathcal{B}_{M-1}$, we can write

$$
s_{n}=\sum_{m=0}^{M-1} g_{m} \prod_{b_{n}^{(i)} \in \mathcal{B}_{m}} b_{n}^{(i)}, \quad n=0,1, \ldots, M-1
$$

which corresponds to a system of $M$ equations (one for each $s_{n}$ and $M$ unknown variables $g_{m}$ ). Using the corresponding binary representation of $m$ with $\mu$ bits, i.e., $m=\left(\gamma_{(\mu-1, m)}, \gamma_{(\mu-2, m)}, \ldots, \gamma_{(1, m)}, \gamma_{(0, m)}\right)$ and defining $\mathcal{B}_{m}$ as the set of bits where the bit $b_{n}^{(i)}$ is included if and only $\gamma_{(i, m)}$ is 1 , we may write

$$
s_{n}=\sum_{m=0}^{M-1} g_{m} \prod_{i=0}^{\mu-1}\left(b_{n}^{(i)}\right)^{\gamma_{(i, m)}} .
$$

The transmitter layered structure shown in Fig. 1 takes advantage of this decomposition in BPSK or QPSK components, and uses a mMIMO scheme with $N_{v} \times N_{m} \times N_{b}$ antenna elements, arranged in $N_{v}$ sets of $T=N_{m} \times N_{b}$ antennas. Conventional beamforming schemes are achieved by a layer 2 with $N_{b}$ antenna elements connected to each amplification branch.

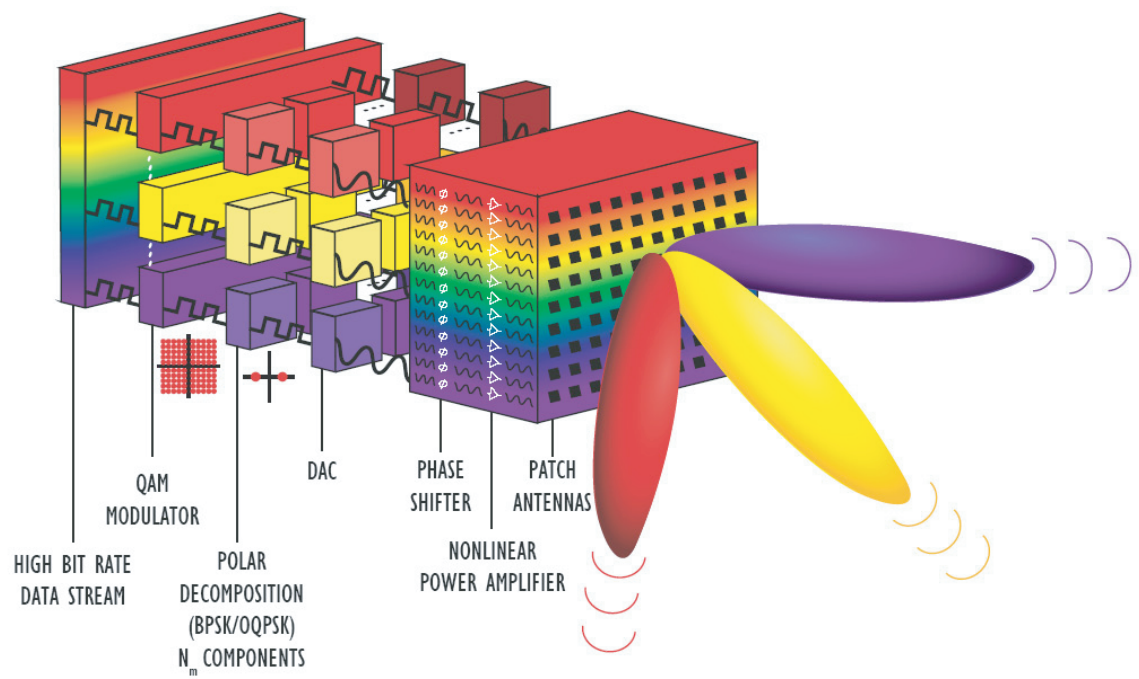

Figure 1: Transmitter layered structure.

Spatial multiplexing is implemented by layer 3 with $N_{v} \times T$ antennas, where the $T$ antennas of layers 1 and 2 are associated to the beams of signal components of the constellation symbol and the $N_{v}$ sets of $T$ antennas are used to transmit simultaneously $N_{v}$ different constellation symbols 
(an example of a system with users using layer 1 and layer 2 combination can be seen in Fig. 2). It follows that power efficiency comes improved due the lower PAPR of the component signals and the possible use of NL amplifiers [4,5]. Thus we may say that layer 1 minimizes problems that can result from nonlinear distortion effects when high order constellations are employed in transmission. The output of each branch of layer 1 is connected to an antenna, being the constellation symbol obtained at channel level through the sum of all the transmitted signals components. Another important aspect lies on the fact that each radio frequency $(\mathrm{RF})$ chain transmits uncorrelated signals, which means an omnidirectional radiation pattern for each set of $N_{m}$ antennas. However, each set of $N_{m}$ antennas implements directivity at information level, since the constellation points of the transmitted signal maintain their positions at the desired direction $\Theta$ but suffer distortion in other directions. Under these conditions, both physical security and power amplification efficiency could be attainable by this transmission structure.

\section{SYSTEM CHARACTERIZATION}

Let us consider the mMIMO scenario shown in Fig. 3 characterized by a point-to-point communication link between $N_{v}$ users, where each user has $T=N_{b} \times N_{m}$ antennas and a base station (BS) acting as receiver with $R \geq N_{v} \times T$ antennas. Each user has the transmitter layered configuration shown in Fig. 2, were Layer 2 is composed by $N_{m}$ sets of $N_{b}$ antennas, each one connected to each amplification branch (for a decomposition of 16-quadrature amplitude modulation (16-QAM) into QPSK or BPSK components we have $N_{m}=2$ and $N_{m}=4$ respectively). Thus, each user has a second layer with $T=N_{b} \times N_{m}$ antennas that transmits simultaneously $N_{m}$ symbol components (more exactly the QPSK or BPSK components from a 16-QAM constellation) with all the beams pointed to the same direction.

With the advent of millimeter waves it is expected an increase in the the number of antennas in the mobile terminals. However, it seems unrealistic to think that this number could be higher than ten, which leads us to assume a maximum number of 12 antennas in each mobile user $(T=12$ corresponds to a layer 2 with arrays of $N_{b}=4$ antennas and a layer 1 with $N_{m}=4$ branches. With QPSK components we have $T=6$ ).

To avoid distortion of the signal that results from the sum of all components at channel level, all the beams related with the signal components should be directed in same direction. Under these conditions it will be crucial to assess the impact of impairments in the alignment of beams carrying

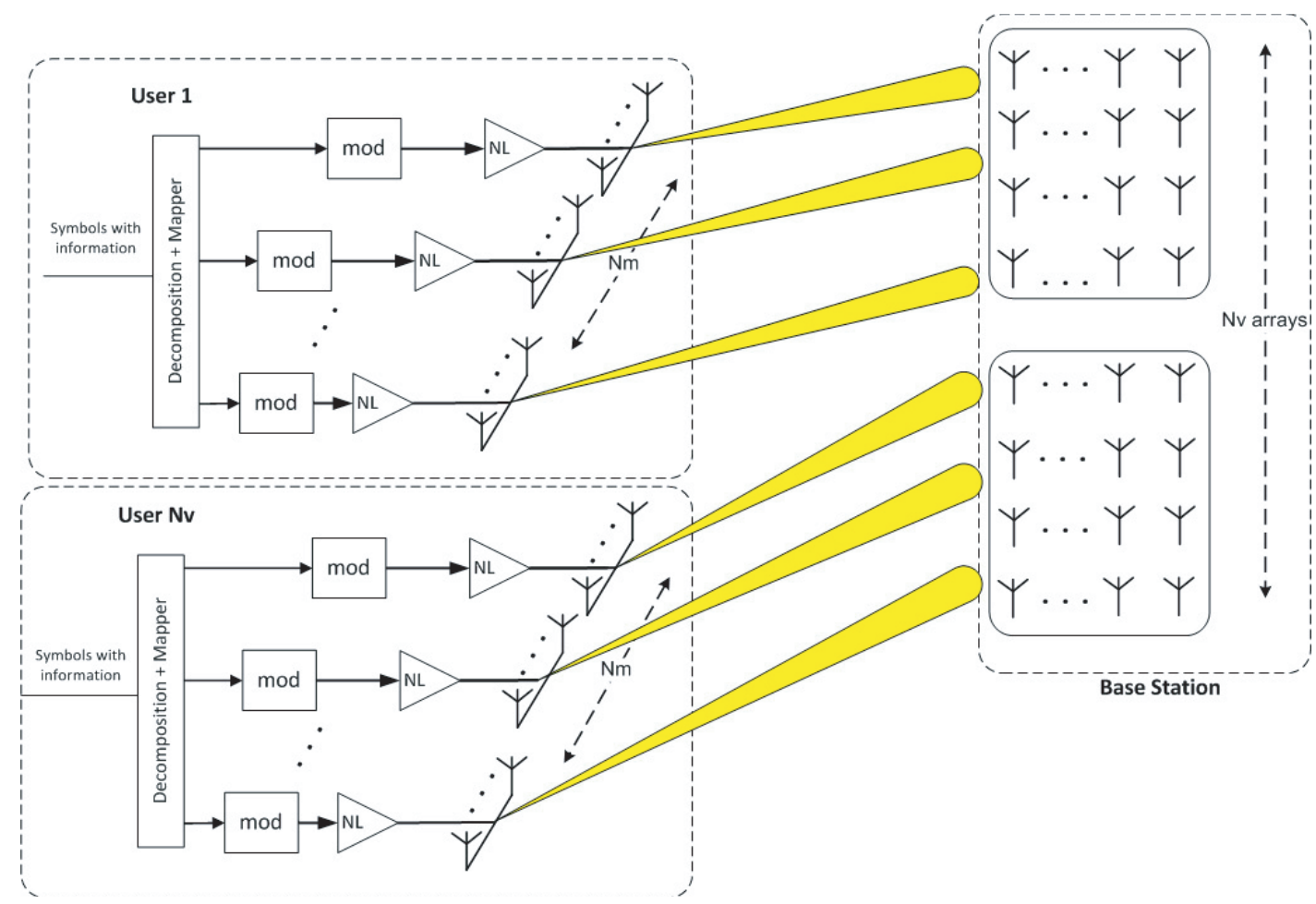

Figure 2: Layered transmitter structure with layer 1 and layer 2. 

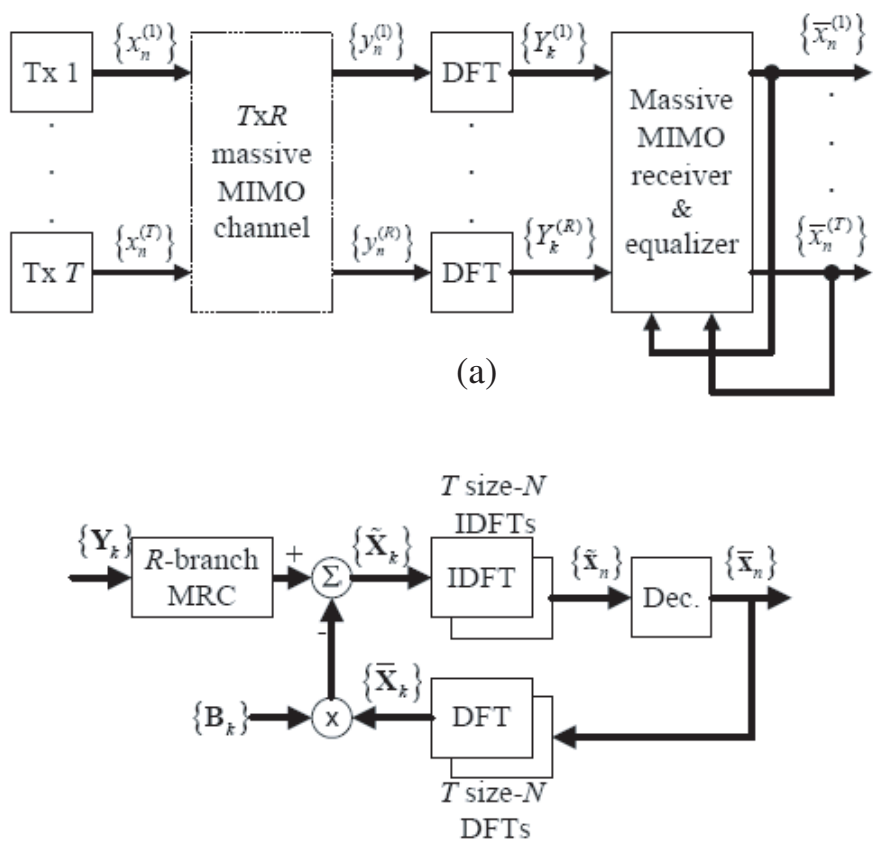

(b)

Figure 3: (a) Overall massive MIMO system for SC-FDE schemes and (b) detail of the massive MIMO receiver and equalization.

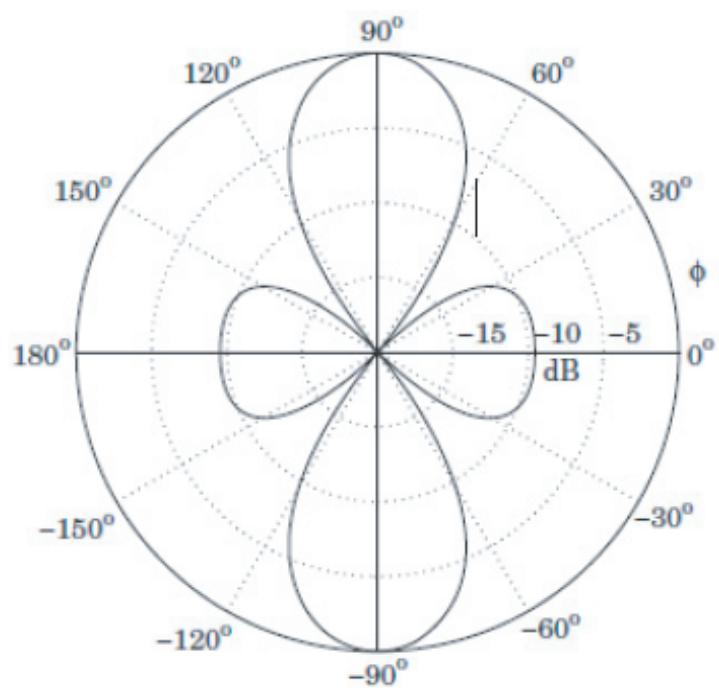

Figure 4: Azimuthal gains of three-element isotropic array with $d=\lambda$ and array weights $\mathbf{a}=[1,1,1]$.

the signal components associated to each user.

No directional interferences are considered among users, since the distance between them leads to an angular separation of $30^{\circ}<\Delta \Theta<55^{\circ}$ which are the azimuthal directions of the radiation beams' nulls, as shown in Fig. 4. Despite the null interference among users, misalignments can occur between the beams associated to each user. As consequence, the several signals components are combined with attenuations that lead to an amplitude distortion of the resulting signal. In simulation results presented further ahead it is assumed misalignments of $10^{\circ}, 13^{\circ}$ and $32^{\circ}$ degrees that correspond to an attenuation of 1,2 and $5 \mathrm{~dB}$, respectively. It is also assumed that only one component is affected, i.e., the stronger or the weaker but not both at same time. For comparison purposes, it will be also considered a scenario without any misalignment between beams, which means that all beams are steered to a specified direction $\Theta$. Coupling effects among antennas are 
avoided by a horizontal spacing of $\lambda$ between antennas of layer 2 and a vertical spacing of $\lambda$ between sets of $N_{m}$ antennas.

As stated before, to deal with channel's frequency selectivity it can be adopted a SC-FDE block transmission technique with a iterative block decision feedback equalization (IB-DFE) receiver whose structure is depicted in Fig. 3(b) [10,11].

At each user the $t$ th antenna array sends the block of $N$ data symbols $\left\{x_{n}^{(t)} ; n=0,1, \ldots, N-1\right\}$ being $\left\{y_{n}^{(r)} ; n=0,1, \ldots, N-1\right\}$ the received block at the $r$ th receiver's antenna. Since a cyclic prefix with a length higher than the overall channel impulse response is appended to each transmitted block and removed at the receiver, the corresponding frequency-domain received block $\left\{Y_{k}^{(r)} ; k=\right.$ $0,1, \ldots, N-1\}$ is given by

$$
\mathbf{Y}_{k}=\left[Y_{k}^{(1)} \ldots Y_{k}^{(R)}\right]^{T}=\mathbf{H}_{k} \mathbf{X}_{k}+\mathbf{N}_{k},
$$

where $\mathbf{H}_{k}$ denotes the $R \times T$ channel matrix for the $k$ th frequency, with $(r, t)$ th element $H_{k}^{(r, t)}$, $\mathbf{X}_{k}=\left[X_{k}^{(1)} \ldots X_{k}^{(T)}\right]^{T}$ and $\mathbf{N}_{k}$ denotes the channel noise.

For an iterative minimum mean squared error (MMSE) receiver the data symbols for a given iteration can be obtained from the inverse discrete Fourier transform (IDFT) of the block $\left\{\tilde{x}_{n}^{(t)} ; n=\right.$ $0,1, \ldots, N-1\}$, given by

$$
\tilde{\mathbf{X}}_{k}=\left[\tilde{X}_{k}^{1} \ldots \tilde{X}_{k}^{(R)}\right]^{T}=\mathbf{F}_{k} \mathbf{Y}_{k}-\mathbf{B}_{k} \overline{\mathbf{X}}_{k},
$$

(more details can be seen in [12]), where $\mathbf{I}$ is an identity matrix and $\alpha=E\left[\left|N_{k}^{(r)}\right|^{2}\right] / E\left[\left|X_{k}^{(t)}\right|^{2}\right]$ is assumed identical for all antennas $t$ and $r, F_{k}$ is the feed forward coefficient, $B_{k}$ is the backward coefficient and $\rho$ denotes the correlation coefficient that can be computed as described in $[9,11,12]$. Interference cancelation is done using $\overline{\mathbf{X}}_{k}=\left[\bar{X}_{0} \ldots \bar{X}_{N-1}\right]$, with $\bar{X}_{k}$ denoting the frequency-domain average values conditioned to the FDE output for the previous iteration, which can be computed as described in $[12,13]$. Obviously, in first iteration this receiver is equivalent to a linear frequencydomain MMSE receiver since no information is available about the transmitted symbols and $\overline{\mathbf{X}}_{k}=\mathbf{0}$. Subsequent iterations employ the average values conditioned to the receiver output from previous iteration to remove the residual intersymbol interference (ISI) and inter user interference.

To avoid the IB-DFE's computational complexity of $O\left(N_{R}^{3}\right)$ (due to channel matrix inversions in each iteration), two low-complexity iterative frequency-domain receivers, denoted as maximum ratio detection (MRD) and equal gain detection (EGD) are also considered as in [14]. Also, having in mind previous results from [14], regarding the influence on performance the ratios $R / T$ between receiving and transmitting antennas, it is assumed $R / T>4$ in the simulations presented further on.

As mentioned in [14], the MRD receiver is characterized by

$$
\tilde{\mathbf{X}}_{k}=\mathbf{\Psi} \mathbf{H}_{k}^{H} \mathbf{Y}_{k}-\mathbf{B}_{k} \overline{\mathbf{X}}_{k},
$$

and

$$
\mathbf{B}_{k}=\mathbf{\Psi} \mathbf{H}_{k}^{H} \mathbf{H}_{k}-\mathbf{I}
$$

where $\boldsymbol{\Psi}$ denotes a diagonal matrix whose $(t, t)$ th element is given by $\left(\sum_{k=0}^{N-1} \sum_{r=1}^{R}\left|H_{k}^{(r, t)}\right|^{2}\right)^{-1}$, takes advantage of the fact that $\mathbf{H}_{k}^{H} \mathbf{H}_{k} \approx R \mathbf{I}$, which is accurate when both conditions are met: $R \gg 1$ and small correlation between different channels. The EGD is characterized by

$$
\mathbf{B}_{k}=\mathbf{\Psi} \mathbf{A}_{k}^{H} \mathbf{H}_{k}-\mathbf{I}
$$

which takes advantage from the fact that the matrix elements outside the main diagonal of $\mathbf{A}_{k}^{H} \mathbf{H}_{k}$, are much lower than the ones form the main diagonal, where $\left(i, i^{\prime}\right)$ th element of the matrix $\mathbf{A}$ is $[\mathbf{A}]_{i, l}=\exp \left(j \arg \left([\mathbf{A}]_{i, l}\right)\right)$, when $R \gg 1$ and small correlation exists between channels associated to different transmit and receive antennas.

\section{PERFORMANCE ASSESSMENT}

In this section we present the performance of the proposed system that accounts effects of imperfect beam alignment. All simulation results are based on Monte Carlo simulations with a stop criteria 
of 100 error events where considered to obtain the average bit error rate (BER) for a Rayleigh channel. Two different configurations are considered for layer 1: in the first one the 16-QAM symbol is decomposed into $N_{m}=2$ QPSK components and in the second one the 16-QAM symbols is represented as a combination of $N_{m}=4$ BPSK components. Layer 2 is implemented by $N_{m}$ uniform phased arrays with 3 antennas and the radiation pattern of Fig. 4. Misalignments $\Delta \Theta$ may be $10^{\circ}, 13^{\circ}$ and $33^{\circ}$ and may affect the weakest or strongest component, but not both at same time. Also, a value of 10 is adopted for the ratio $R / T$ (as seen in previous results this ratio assures better performance). It is also assumed linear power amplification at the transmitter, perfect channel estimation and perfect synchronization conditions at the receiver. A maximum of 4 iterations is considered at the receiver (previous simulations with iterations until 10 showed that after 4 the improvements in performance do not justify the increase on receiver's complexity). All BER results are expressed as function of $\frac{E_{b}}{N_{0}}$, where $N_{0} / 2$ denotes the noise variance and $E_{b}$ represents the energy of the transmitted bits.

Results shown in Figs. 5, 6 and 7 refer IB-DFE, MRD and EGD receivers with similar simulation conditions and system configurations based on combination of layer 1 and layer 2. From the results of Figs. 5 and 6 it is also clear that both IB-DFE and MRD receivers have similar performances. As expected, these two receivers perform better than the EGD. Also, the results for $\Delta \Theta=0^{\circ}$

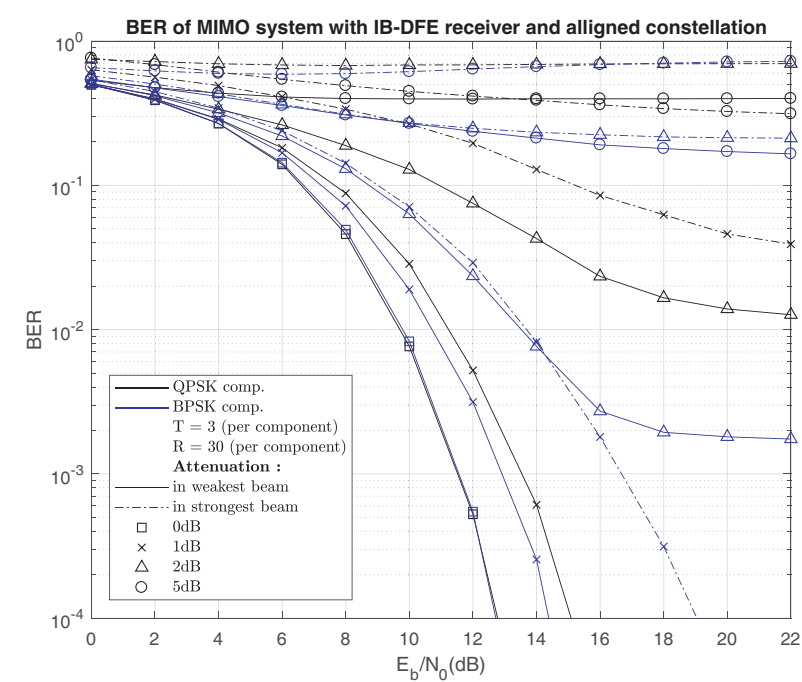

Figure 5: BER performance for IB-DFE and $R / T=$ 10 .

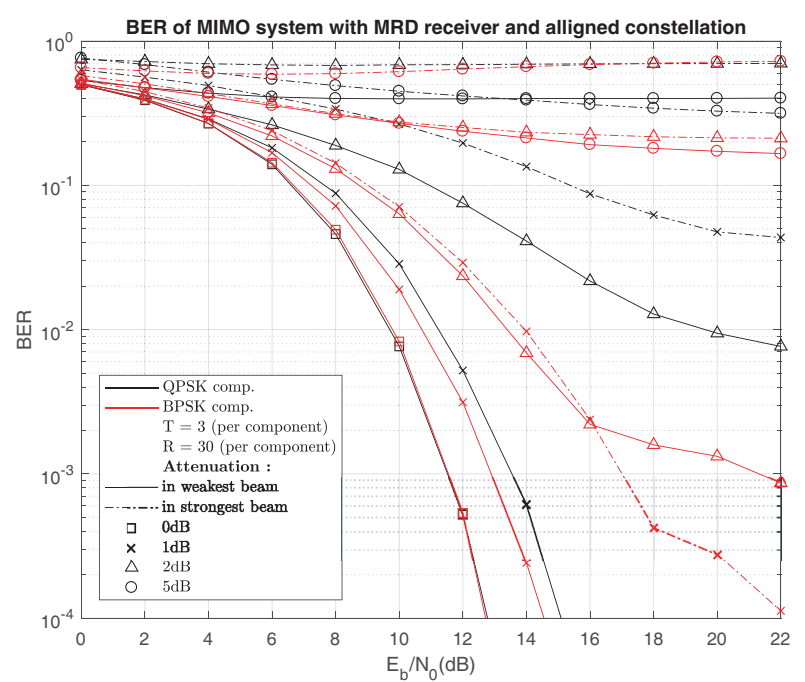

Figure 6: BER performance for MRD receiver and $R / T=10$

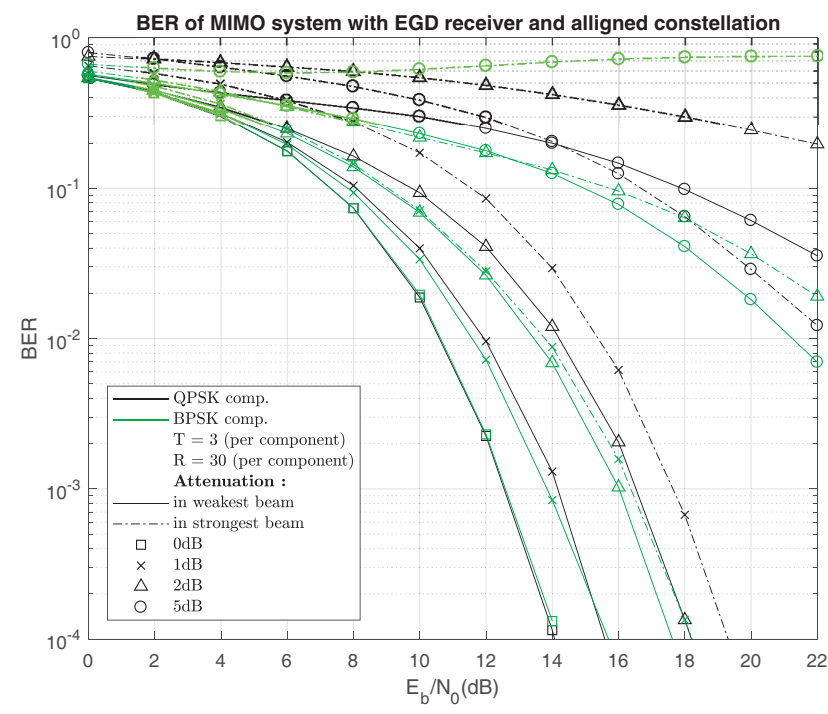

Figure 7: BER performance for EGD receiver and $R / T=10$. 
are similar to the ones obtained by a transmitter using only Layer 1 or by a conventional spatial multiplexing scheme, which means that combination of layer 1 and layer 2 does not sacrifice system's performance when misalignments are nonexistent.

It can be seen that misalignments $\Delta \Theta \leq 10^{\circ}$ on the the weakest beam component have low impact in system performance (around $1 \mathrm{~dB}$ for a BER of $10^{-4}$ for $N_{m}=4$ and $1.5 \mathrm{~dB}$ for $N_{m}=2$ ). In perfect alignment conditions the EGD performance has a degradation of $1.5 \mathrm{~dB}$ (for a BER of $10^{-4}$ ) compared with the other two receivers. However, this is not the case of the strongest beam where the performance degradation of both receivers IB-DFE and MRD is near to $5.5 \mathrm{dBs}$, even for a misalignment of $\Delta \Theta=10^{\circ}$. In similar conditions the EGD has a degradation of $5 \mathrm{dBs}$ (for a BER of $10^{-4}$ ). Also, for misalignments of the stronger component higher than $\Delta \Theta=10^{\circ}$ both MRD and EGD are more severely affected than the EGD receiver (this behavior can be easily seen by comparing the results an attenuation of $2 \mathrm{~dB}$ of the stronger component). However, for misalignments with attenuations higher than $2 \mathrm{~dB}$ this higher receiver's sensitivity compromises the decoding capacity of the system based on IB-DFE and MRD receivers.

As expected attenuations due to misalignments of the QPSK components have a stronger effect on the resulting constellation than attenuations in the BPSK component. This behavior is justified by the fact that attenuations affecting QPSK components affects at same time two BPSK components, and consequently have a stronger distortion effect in the symbols resulting from the combination of the components. The cause for this behavior can be seen in Figs. 8 and 9 where it is obvious the higher distortion due to QPSK beams misalignments.

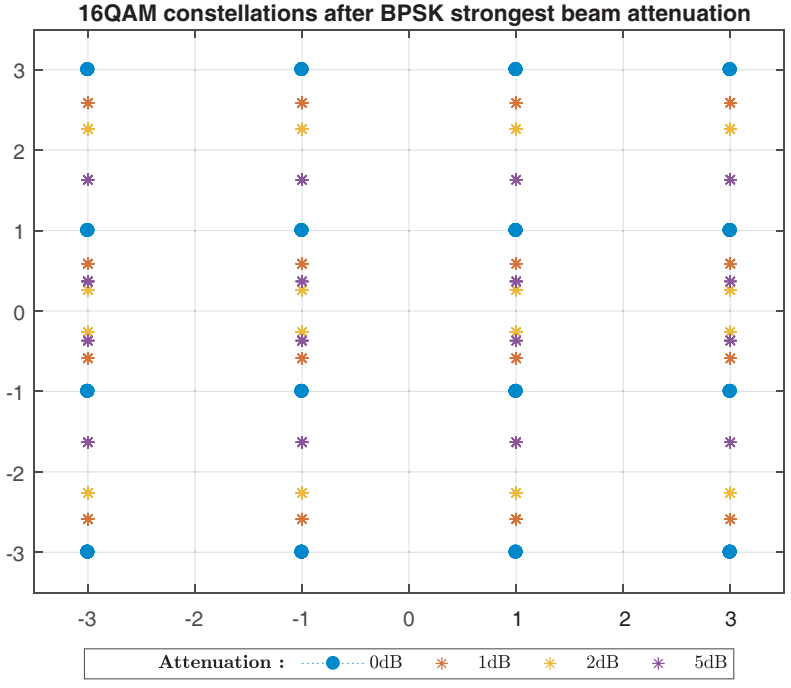

Figure 8: Constellation distortion with misalignments of the strongest BPSK component.

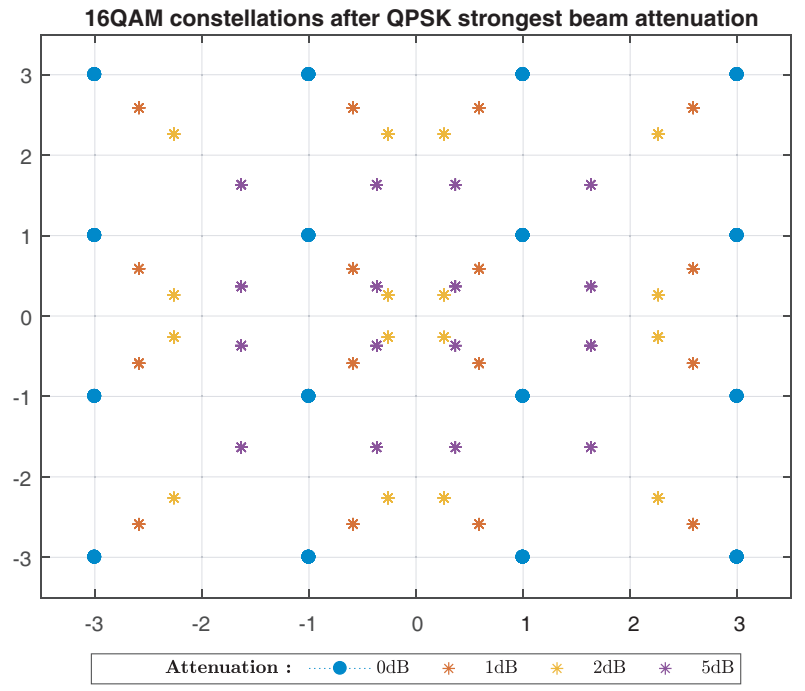

Figure 9: Constellation distortion with misalignments of the strongest QPSK component.

It is important to do a remark about the physical security level assured by this combination of layers. Under perfect alignment conditions the security level is similar to the systems employing only layer 1 or combinations of layer 1 and layer 3 (previous analysis concerning the security have shown that the use of Layer 2 for beamforming has no negative impact on the security level assured by the system. Moreover, under perfect alignment conditions security can even increase due to the energy separation between users). It should be mentioned that the sensitivity to any angular separation between users due to constellation shaping becomes reinforced to the energy separation achieved by the beamforming, and layer 2 never affects negatively the security level due constellation shaping performed by layer 1 .

\section{CONCLUSION}

In this paper it was shown that a transmitter based on a double layer structure with information directivity and beamforming could be employed with multilevel constellations to improve power amplification efficiency and to assure physical security. Results show the low tolerance of performance against attenuations of higher than $1 \mathrm{~dB}$ on the strongest component, regardless the type of receiver. However, the system shows good tolerance against beams misalignments since misalignments $\Delta \Theta$ should be higher than $13^{\circ}$ and affect the strongest component to have a clear impact in 
system performance. Despite this behavior the system performance has a good tolerance margin against misalignments $\Delta \Theta \leq 10^{\circ}$ affecting the weaker or the strongest beam. This behavior let us to conclude that beamforming achieved by layer 2 could be employed together with layer 1 .

\section{ACKNOWLEDGMENT}

This work was supported in part by IT UID/EEA/50008/2013 (plurianual founding and project GLANCES), Foundation for Science and Technology (PhD scholarship SFRH/BD/131093/2017), EnAcoMIMOCo EXPL/EEI-TEL/2408/2013, UID/EEA/50008/2013 — MM5G and and project PES3N (Ref: POCI-01-0145-FEDER-030629).

\section{REFERENCES}

1. Boccardi, F., R. Heath, A. Lozano, T. Marzetta, and P. Popovski, "Five disruptive technology directions for 5G," IEEE Communications Magazine, Vol. 52, No. 2, 74-80, Feb. 2014.

2. Busari, S. A., K. Huq, S. M. Mumtaz, L. Dai, and J. Rodriguez, "Millimeter-wave massive MIMO communication for future wireless systems: A survey," IEEE Communications Surveys and Tutorials, Vol. 20, No. 2, 836-869, 2017.

3. Montezuma, P. and R. Dinis, "Implementing physical layer security using transmitters with constellation shaping," Proc. IEEE ICCCN 2015, Las Vegas, NV, US, Aug. 2015.

4. Astucia, V., P. Montezuma, R. Dinis, and M. Beko, "On the use of multiple grossly nonlinear amplifiers for higly efficient linear amplification of multilevel constellations," Proc. IEEE VTC 2013-Fall, Las Vegas, NV, US, Sep. 2013.

5. Montezuma, P. and A. Gusmão, "Design of TC-OQAM schemes using a generalised nonlinear OQPSK-type format," IEE Elect. Letters, Vol. 35, No. 11, 860-861, May 1999.

6. Falconer, D., S. Ariyavisitakul, A. Benyamin-Seeyar, and B. Eidson, "Frequency domain equalization for single-carrier broadband wireless systems," IEEE Comm. Mag., Vol. 4, No. 4, 58-66, Apr. 2002.

7. Gusmão, A., R. Dinis, J. Conceicão, and N. Esteves, "Comparison of two modulation choices for broadband wireless communications," IEEE VTC 2000 (Spring), Vol. 2, May 2000.

8. Ferreira, A., G. Gaspar, P. Montezuma, and R. Dinis, "Combining info and spatial directivities in multiple antenna transmission systems," IEEE YEF-ECE 2017, Costa da Caprica, May 2017.

9. Dinis, R., P. Montezuma, N. Souto, and J. Silva, "Iterative frequency-domain equalization for general constellations," IEEE Sarnoff Symposium, Princeton, USA, Apr. 2010.

10. Benvenuto, N. and S. Tomasin, "Block iterative DFE for single carrier modulation," IEE Elec. Let., Vol. 39, No. 19, 1144-1145, Sep. 2002.

11. Dinis, R., R. Kalbasi, D. Falconer, and A. Banihashemi, "Iterative layered space-time receivers for single-carrier transmission over severe time-dispersive channels," IEEE Comm. Letters, Vol. 8, No. 9, 579-581, Sep. 2004.

12. Silva, P. and R. Dinis, Frequency-domain Multiuser Detection for CDMA Systems, River Publishers, Aalborg, 2012.

13. Silva, F., R. Dinis, and P. Montezuma, "Estimation of the feedback reliability for IB-DFE receivers," ISRN Communications and Networking, Vol. 2011, Article No. 30, Jan. 2011.

14. Borges, D., P. Montezuma, and R. Dinis, "Low complexity MRC and EGC based receivers for SC-FDE modulations with massive MIMO schemes," 2016 IEEE GlobalSip, Washington, DC, USA, Dec. 2016.

15. Borges, D., P. Montezuma, and R. Dinis, "Low complexity MRC and EGC based receivers for SC-FDE modulations with massive MIMO schemes," 2016 IEEE GlobalSip, Washington, DC, USA, Dec. 2016. 